\title{
Pigment Epithelium-Derived Factor: Chemistry, Structure, Biology, and Applications
}

\author{
S. Patricia Becerra, ${ }^{1}$ Crispin R. Dass, ${ }^{2}$ Takeshi Yabe, ${ }^{3}$ and Susan E. Crawford ${ }^{4}$ \\ ${ }^{1}$ Section of Protein Structure and Function, Laboratory of Retinal Cell and Molecular Biology, National Eye Institute, \\ Bethesda, MD 20892, USA \\ ${ }^{2}$ School of Biomedical Sciences, Victoria University, Melbourne, VIC, Australia \\ ${ }^{3}$ Department of Medical Pharmacy, Setsunan University, Osaka 573-0101, Japan \\ ${ }^{4}$ Department of Pathology, St. Louis University, St. Louis, MO 63104, USA
}

Correspondence should be addressed to S. Patricia Becerra, becerrap@nei.nih.gov

Received 27 August 2012; Accepted 27 August 2012

Copyright (c) 2012 S. Patricia Becerra et al. This is an open access article distributed under the Creative Commons Attribution License, which permits unrestricted use, distribution, and reproduction in any medium, provided the original work is properly cited.

Pigment epithelium-derived factor (PEDF) is a multifunctional serpin protein with demonstrable neurotrophic, antiangiogenic, antitumorigenic, antimetastatic, antiinflammatory, antioxidative properties among others. PEDF exists naturally in most organs of the human body, and it is released from most cell types as an extracellular diffusible and circulating glycoprotein. It has been studied mostly in the eye, where its levels are altered in diseases characterized by retinopathies, such as age-related macular degeneration and diabetic retinopathy. The importance of PEDF in the development, maintenance, and function of the retina and CNS is evident in animal models for inherited and lightinduced retinal degeneration, as well as for degeneration of spinal cord motor neurons. Pathological ocular neovascularization- and retinal degeneration-related animal models have prompted clinical development. Clinical trials to assess the safety of a viral expression vector for PEDF in the context of age-related macular degeneration have been performed. Interestingly, PEDF has multiple biological effects against tumors, and its efficacy has been demonstrated in several animal models for tumor growth and progression. The mechanisms of PEDF action on tumors have been associated to inhibition of tumor angiogenesis, and also negative effects directed on tumor cells. Recently, the involvement of PEDF in stem cell biology has been revealed. Moreover, PEDF is a potential diagnostic tool for several diseases triggered by pathological neovascularization, retinal degenerations, or tumors.
The present issue includes three original research and four review articles. In one article, V. Chandolu and C. R. Dass review the biological functions of PEDF against cancer, with a focus on a particular type of bone cancer called osteosarcoma. They summarize the progress in understanding the function of PEDF in antiangiogenesis, tumor cell differentiation, and direct tumor cell inhibition. In another review article, J.-T. Liu et al. address the role of PEDF in stem/progenitor cell-associated neovascularization, in particular in cardiovascular and neurovascular biology. M. Elahy et al. review the promising significance of PEDF in stem cell biology, specifically in human embryonic stem cells, mesenchymal stem cells (MSCs), neural stem sells (NSCs), and stem cells overexpressing the PEDF gene. X.-F. Zhu et al. summarize the advances of PEDF in diabetic retinopathy, its protective effect on oxidative stress, which is the main trigger for the pathology, and the potential application of PEDF in diabetic retinopathy. In a research article, P. Subramanian et al. report the identification of biochemical forms of PEDF with distinct biological effects on tumor cells, which may explain the multifunctional modality of this protein. In a second research article, M. L. Broadhead et al. report the effects of two PEDF-derived peptides on a clinically relevant murine model of osteosarcoma with spontaneous metastasis. Finally, T. Falk et al. report that human retinal pigment epithelial cells grown on plastic-based microcarriers retain the ability to produce both PEDF and vascular endothelial growth factor VEGF-A. This constitutes a novel candidate 
delivery system for neurotrophic factors with potential application in neurodegenerative diseases, such as Parkinson's.

In summary, given the multimodal nature of PEDF, the present issue aims to enhance our understanding of the chemistry, structure, biology, and application of PEDF in biomedicine. It gives an overview of the current status of research on PEDF and will prove useful as source of reference for students and researchers.

S. Patricia Becerra

Crispin R. Dass

Takeshi Yabe

Susan E. Crawford 

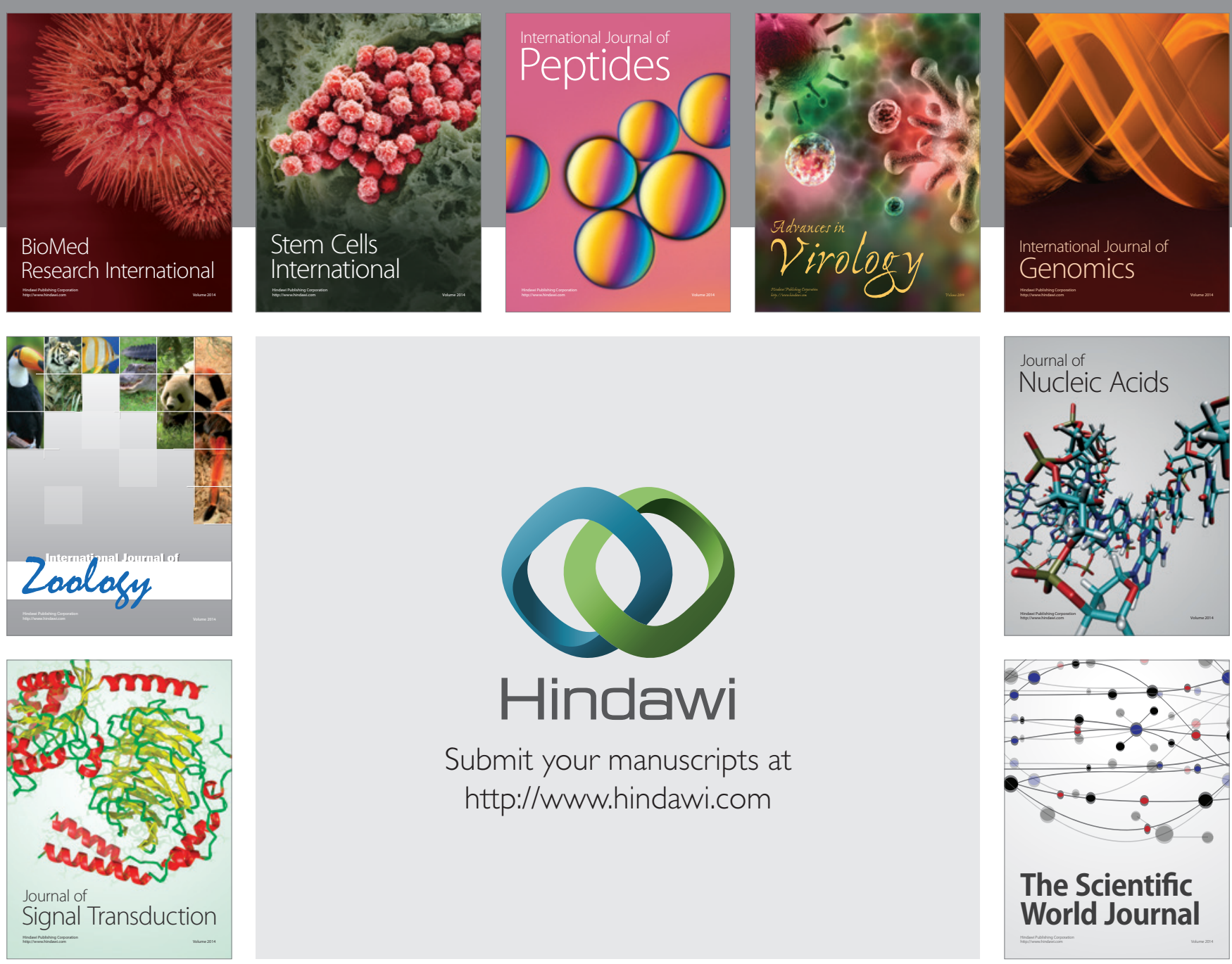

Submit your manuscripts at

http://www.hindawi.com
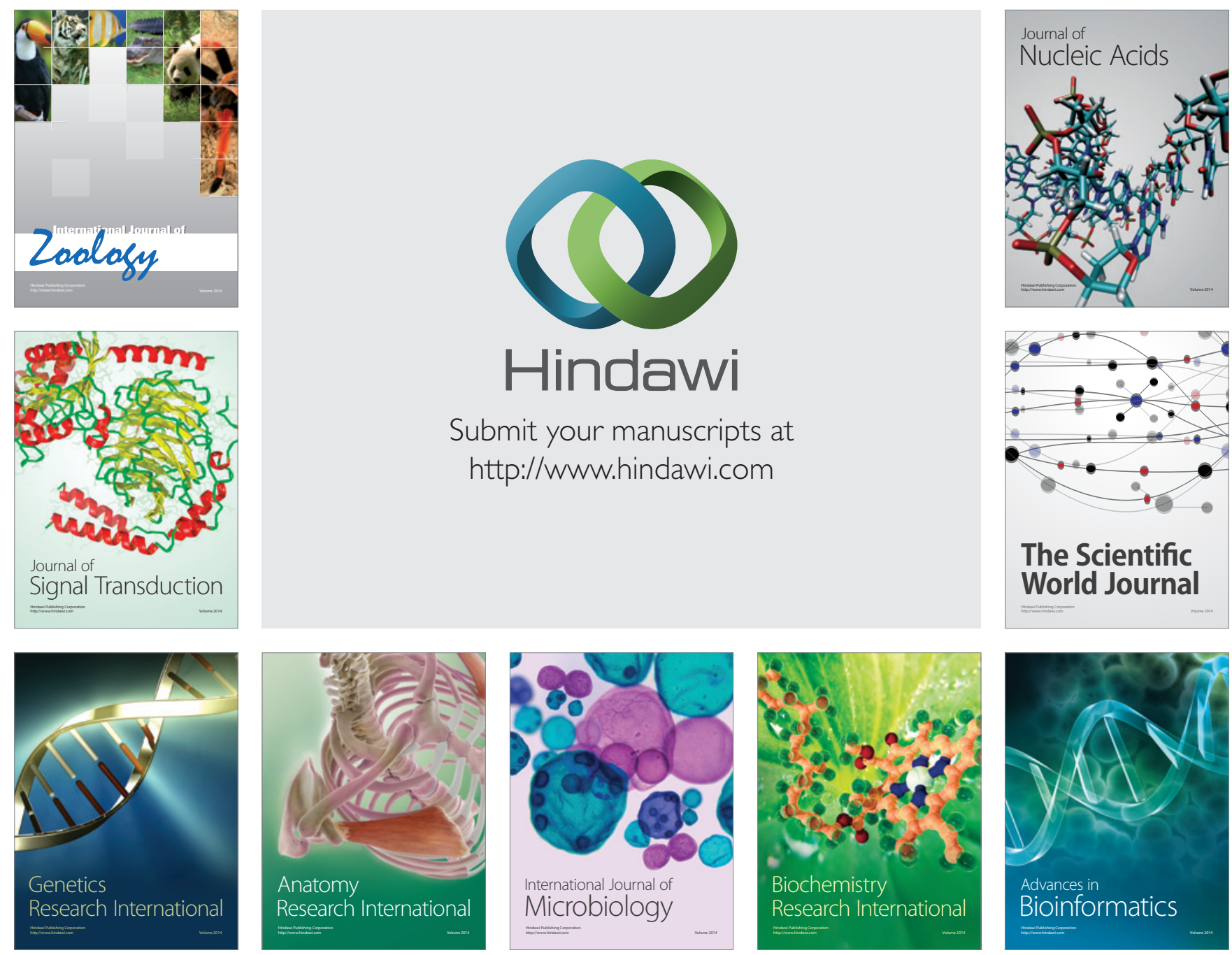

The Scientific World Journal
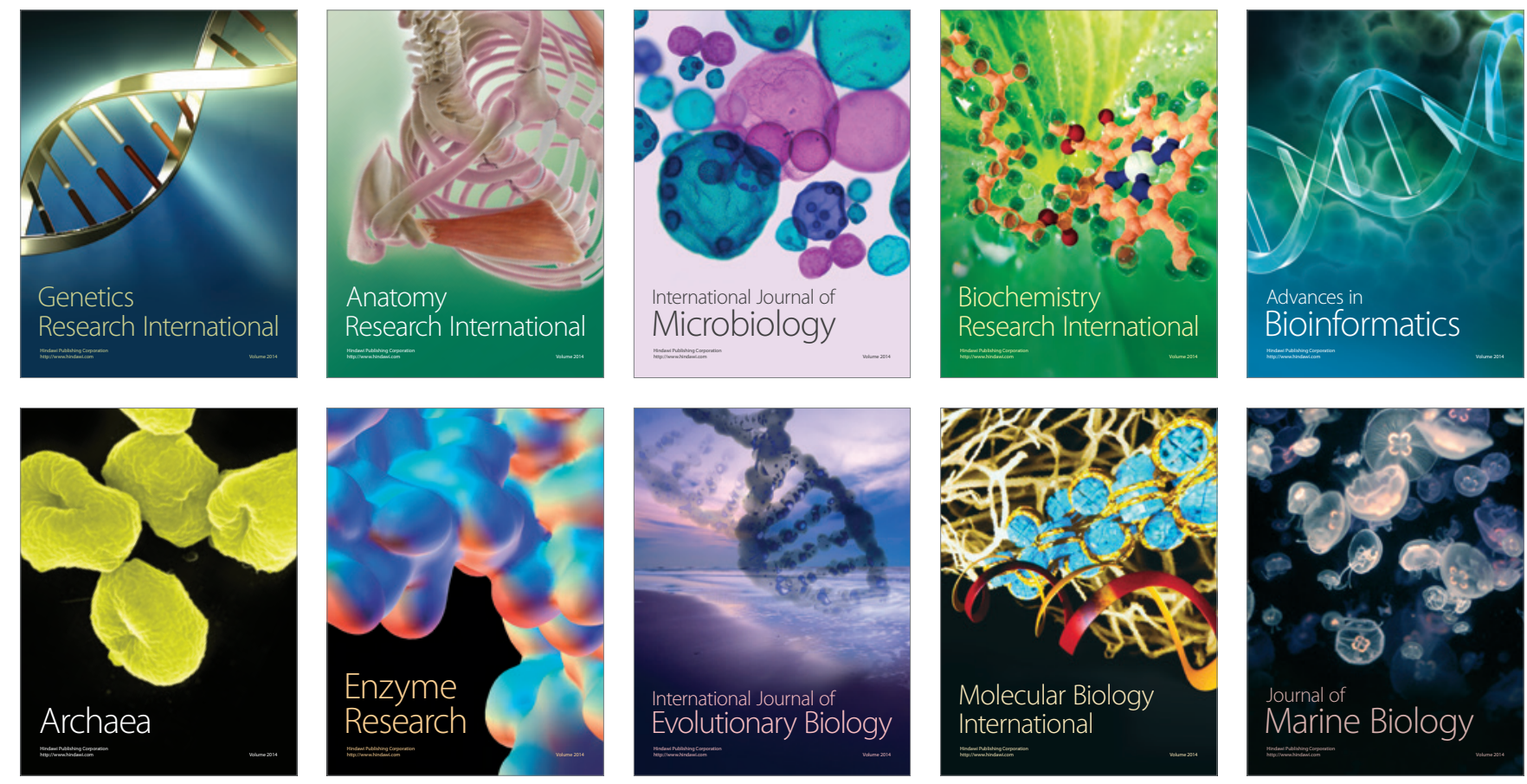